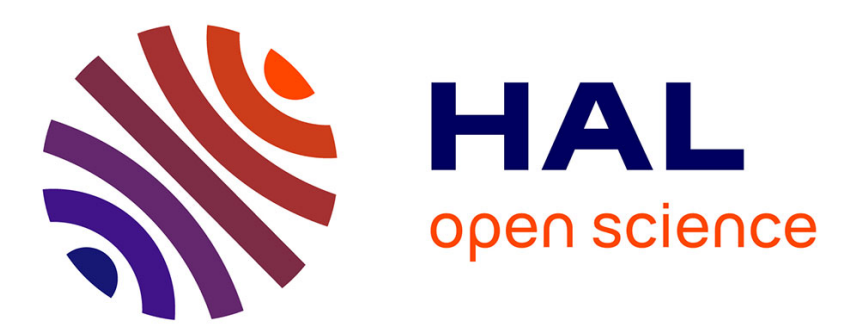

\title{
Efficacy and efficiency indexes for a multi-criteria industrial performance synthesized by Choquet integral aggregation
}

Lamia Berrah, Gilles Mauris, Jacky Montmain, Vincent Clivillé

\section{- To cite this version:}

Lamia Berrah, Gilles Mauris, Jacky Montmain, Vincent Clivillé. Efficacy and efficiency indexes for a multi-criteria industrial performance synthesized by Choquet integral aggregation. International Journal of Computer Integrated Manufacturing, 2008, 21 (4), pp.415-425. 10.1080/09511920701574255 . hal-00353853

\section{HAL Id: hal-00353853 \\ https://hal.science/hal-00353853}

Submitted on 7 Jun 2021

HAL is a multi-disciplinary open access archive for the deposit and dissemination of scientific research documents, whether they are published or not. The documents may come from teaching and research institutions in France or abroad, or from public or private research centers.
L'archive ouverte pluridisciplinaire HAL, est destinée au dépôt et à la diffusion de documents scientifiques de niveau recherche, publiés ou non, émanant des établissements d'enseignement et de recherche français ou étrangers, des laboratoires publics ou privés. 


\title{
Efficacy and efficiency indexes for a multi-criteria industrial performance synthesized by Choquet integral aggregation
}

\author{
L. BERRAH $\dagger$, G. MAURIS $\dagger$, J. MONTMAIN $\dagger$ and V. CLIVILLE \\ $\dagger$ LISTIC, ESIA-Université de Savoie, BP 806, F-74016 Annecy cedex, France \\ †LGI2P, Ecole des Mines d'Alès, Site EERIE, Parc Scientifique G. Besse, \\ F-30035 Nîmes, France
}

\begin{abstract}
Nowadays, despite widespread recognition of the importance of performance measurement systems (PMSs), there are some issues that require further investigations if PMSs are to be effective in their role of control support. This article seeks to illustrate that the Choquet integral aggregation operators can address the problem of taking interactions between performance criteria into account. But the use of this quite complex approach requires to explicitly define pieces of information aimed at aiding decision makers by a better understanding of the contribution of the elementary performances to the overall one, and at better assessing the different ways of improving the overall performance. In this view, indexes of efficacy and efficiency of the elementary performances aggregated by a 2-additive Choquet integral are proposed and applied to a case submitted by a small and medium-sized company (SME).
\end{abstract}

Keywords: Performance measurement systems PMSs; Performance aggregation, performance analysis; Efficacy and efficiency indexes; 2-additive Choquet integral

\section{Introduction}

In the post-Taylorian organization prevailing in the 19752006 period, the performance expression has progressively become a multi-criteria one, with the integration of the technical criteria such as quality levels and delivery date in addition to conventional costs (Panlan et al. 1992, Lebas 1995, Grabot 1998). In this context, a compromise has to be effected between the satisfaction of these criteria, that influence positively or negatively one another, even if, paradoxally the Taylorian maximization spirit remains a usual ambition for many managers. From this perspective, technical reports on the status of the processes have been introduced (Fortuin 1988, Berrah et al. 2000, Cliville et al. 2004). Moreover, performance depending on the production processes and services, it must be deployed within the various production activities, according to a defined action plan. Therefore, performance expressions are considered not only at the strategic level but at all decision levels (namely, strategic, tactical and operational). Thus, performance expressions, both financial and non financial, must be considered from top to bottom for all the activities or processes to be controlled (Bititci 1995, Rangone 1996, Ghalayini et al. 1997, Suwignjo and Bititci 2000, Cha and Jung 2003).

Therefore, to deal with the complexity of the current industrial context, new diagnosis/control activities which are aimed at driving continuous improvement, have to define, compare and choose action plans with regard to the relationships among performance expressions (Bradford and Childe 2001). This is the purpose of the so-called performance measurement systems (PMSs) Bititci 1995, Neely 1999, Kuent and Krahn 1999). From a global point of view, a PMS is a multi-criteria instrument for informing decision makers about, among other things, the level of performance, the reasons for poor or good performance,

\footnotetext{
*Corresponding author. Email: Lamia.Berrah@univ-savoie.fr
} 
the criteria for which improvement is required. It is made of a set of performance expressions to be consistently organized with respect to the objectives of the company. For instance, the objectives/performances of the manufacturing workshops contribute to objectives/performances of the manufacturing plants, that in turn contribute to objectives/performances of the company. Then in order to support the decision, the set of performances is processed for comparing the different situations. One main approach is to reduce the dimensionality by aggregating the elementary performances, outranking methods are also used but can lead to incomparability of situations. More precisely, the major problems in the design of such systems concern:

(a) on the one hand, the identification of the performance structure by the break-down of the overall objective into elementary ones along organizational levels (strategic, tactical or operational), and the corollary aggregation of the elementary performance expressions to consolidate the value of the overall one (Grabot 1998, Cliville et al. 2004);

(b) on the other hand, in addition to the performances, the definition of the relevant pieces of information to help understand how elementary performances affect the overall one, and to help determine action plans contributing to the performance improvement (Montmain et al. 2004).

Concerning the first point, the main industrial practice generally consists in building a tree structure composed of independent performance criteria and in translating all performance expressions associated with the various heterogeneous criteria into a common reference, (cost or satisfaction degree), and then simply making a weighted mean to obtain the overall performance. In this line, many approaches proposed in the literature are based on the analytic hierarchy process (AHP) method (Rangone 1996, Suwignjo and Bititci 2000). Drawbacks of this approach are that representing the relationships between performance criteria by a simple independent tree structure is not straightforward owing to different types of interactions between criteria (see section 2). This is why we previously proposed, for this purpose, to use Choquet integral aggregation operators (Berrah et al. 2003). The determination of the aggregation parameters is based on a MACBETH (Multi Attractiveness Categorical Based Evaluation TecHnique) (Bana e Costa 2004) inspired methodology that allows to ensure the coherence between the elementary and aggregated performance expressions from experts' preferences.

The second point is little considered in the literature. Indeed, the performance diagnosis and improvement are generally not formally considered as decision makers take decisions in an intuitive manner, the simplicity of the weighted mean making this possible. The interactions between criteria make it more difficult for decision taking to identify the criteria at the origin of a poor overall performance or to be improved in priority to improve the overall performance as much as possible. Therefore, the aim of the present paper is explicitly to define, from a Choquet integral performance aggregation, such pieces of information aimed at aiding to understand the causes of poor overall performance and to determine ways to improve it.

The paper is organized as follows. Section 2 concerns the multi-criteria characteristics of the industrial performance. We will particularly present the 2-additive Choquet integral as an answer to the performance aggregation problem and the MACBETH-inspired method to obtain commensurate quantitative performance expressions. Then, in section 3, quantitative performance diagnosis tools, i.e. efficacy and efficiency indexes, are proposed to support decision makers. Section 4 illustrates the proposed approach by means of a practical case study issued from a small and medium sizedenterprise (SME) that manufactures kitchens, bathrooms and storing spaces.

\section{Multi-criteria performance measurement framework}

Let us recall that in our view, one key role of a performance measurement system is the computation of the elementary performance expressions and their bottom-up aggregation to obtain an overall performance expression.

\subsection{Considerations for coherent performance expressions}

The aggregation approach requires a previous identification of the performance structure by the break-down of the overall objective considered into elementary ones at different organizational levels (strategic, tactical or operational). Generally, this identification is made by human expertise and results in logical relationships between the involved variables (see an example in figure 1).

The two main sub problems concerning the quantification of the performance are:

(a) the expression of quantified performance reflecting the satisfaction of the elementary objectives,

(b) the expression of a quantified performance reflecting the satisfaction of the overall objective knowing that a direct determination is generally not easy to achieve.

Concerning the last point, the most frequently adopted approach is an aggregation based one which allows to synthesize some elementary pieces of information into an overall one. In the context of industrial performance 


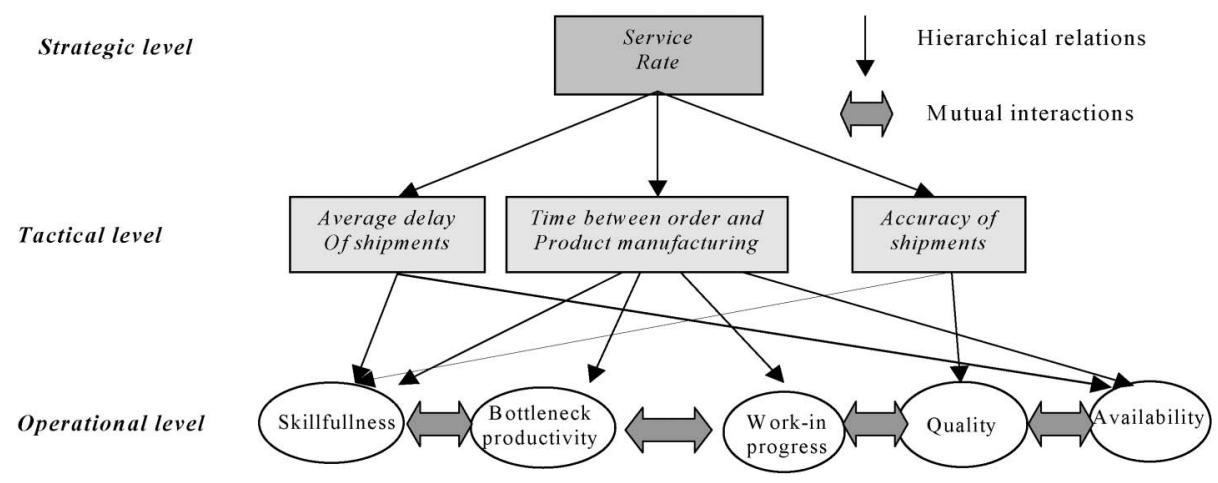

Figure 1. Example of a performance structure.

expression, the aggregation processing can be formalized by the following mapping

$$
\begin{aligned}
A g: & E_{1} \times E_{2} \times \ldots \times E_{n} \rightarrow E \\
& \left(P_{1}, P_{2}, \ldots, P_{n}\right) \rightarrow P_{\text {overall }} \\
& =A g\left(P_{1}, P_{2}, \ldots, P_{n}\right)
\end{aligned}
$$

$E^{i}$ is the universe of discourse of the elementary performance expressions $\left(P_{1}, \ldots, P_{n}\right)$ and $E$ is the universe of discourse of the overall performance expression $P_{\text {overall }}$.

Regarding the information involved in the performance indicators, $P_{i}$ is the elementary performance expression, and $P_{\text {overall }}$ is the aggregated performance expression. In the literature, different kinds of performance expressions are considered depending on how the universes $E_{i}$ are defined, percentage of defects, production time, number of products. So a PMS designer has to consider:

(a) the expression of heterogeneous elementary expressions on the same universe in order to facilitate their subsequent aggregation,

(b) the choice of an aggregation operator and the determination of its parameters.

In the current practice, it is proposed to build normalized elementary expressions on an interval $[0,1]$ without a unit. Different types of comparison operators such as ratios or fuzzy operators are proposed based on expert knowledge. But the subsequent aggregation processing is considered separately from the elementary performance expressions. Thus no global vision allows experts to ensure the coherence between elementary and aggregated expressions.

Concerning the second point, the main aggregation operator is the weighted average mean (WAM) (Bitici 1995). This type of compromise operator is well adapted for independent criteria. In a lot of propositions an expert chooses the weights directly. However in a majority of cases, the weights are determined by AHP using ratio comparison (Saaty 2004, Dasarathy 2001). However, the
WAM operator is not able to take into account synergy, contradictions, redundancy of criteria which are often observed in the current industrial context. In order to determine elementary and aggregated performance expressions coherently, the theory of measurement specifies some requirements, which can be expressed as follows (Labreuche and Grabisch 2003):

(a) the elementary expressions (according to different criteria) have to be defined on the same scale type,

(b) the aggregation operator must be significant for the scale type considered.

Moreover, it is possible to deal with the criteria interaction thanks to the family of the Choquet integral (CI) operators which is an extension of the WAM operator and allows to model different kinds of decision-maker behaviour (Grabisch and Roubens 1996). It has been demonstrated by Labreuche and Gabrisch (2003) that the CI operators are significant for performance expressions defined on an interval scale, for the which the difference is meaningful.

Thus, in summary our approach is:

(a) to express the elementary expressions along the same interval scale $[0,1]$,

(b) to determine the CI parameters in a coherent way.

\subsection{The interval scale definition}

In the current industrial practice, the transformation of physical measures into performance expressions is made according to the following general mapping (Labreuche and Brabisch 2003):

$$
\begin{gathered}
p: \quad O \times M \rightarrow E \\
(o, m) \rightarrow p(o, m)=P
\end{gathered}
$$

$O, M$ and $E$ are respectively the universes of discourse of the set of objectives $o$, of the set of measures $m$ and of the 
performance expression $P$. The key point in differentiating this kind of performance expressions from conventional measurements is in the expression of a satisfaction degree (and not a physical measure), by the comparison of the acquired measures with an objective defined according to the control strategy considered. Generally, the mapping $p$ is a comparison operator such as a distance operator or a similarity operator (Grabisch and Roubens 1996) and the results are expressed within the range $[0,1]$. This approach is useful because it tackles the inter-criteria commensurability issue by considering two common reference points having an absolute meaning for the PMS designer, i.e. 'totally unsatisfying' associated to the performance value 0 , $P=0\left(m=m^{0}\right)$, and 'totally satisfying' associated to the performance value $1, P=1(m=o)$.

But this does not guarantee that the scales obtained are interval ones for the other values of $P$. To achieve this, theoretically, all other values have to be considered. This being difficult, it is possible to consider a limited set of values corresponding to the different situations considered by the decision maker. Indeed, decision-makers have many strategies. They are looking at performance expressions in order to follow the reached improvements. They may also want to determine the best action plan to implement in order to improve the performance. Thus, the elementary performance expressions are defined thanks to semantic judgements concerning a limited set of considered situations in the line of the MACBETH (Multi Attractiveness Categorical Based Evaluation TecHnique) method (Bana e Costa et al. 2004) based on the decision maker's preferences and strengths of preference:

(1) 'A PMS designer prefers a situation $A$ to a situation $B$ ' can be written by $A \succ B$. It means that the performance associated to $A$, noted $P^{A}$ is better than the performance associated to $B$ noted $P^{B}$ which is equivalent: $P^{A}>P^{B}$. If he/she expresses all his/her preferences between the set of situations, he/ she now has an ordinal scale,

(2) 'A PMS designer prefers a situation A to a situation B with a given intensity' can be written by $A \succ^{\text {intensity }} B$. It means that the difference between $P^{A}$ and $P^{B}$ is proportional to this intensity, that is equivalent to $\left(P^{A}-P^{B}\right)=q$ where $q$ represents the intensity. The most frequent proposition for verbal levels of attractiveness for $q$ are (null, very weak, weak, moderate, strong, very strong, extreme). They can be translated as numerical levels $(0,1,2$, $3,4,5,6)[22]$.

If he/she expresses all his/her strengths of preference between the set of situations, he/she now has a cardinal scale, more precisely an interval scale for these values. For other ones, a linear interpolation is made from these reference values. The interval scale $[0,1]$ thus generated is in accordance with the measurement theory. Note that another scale derived form by a linear transformation (defined by two parameters: slope and constant) can also be used.

Example of interval scale definition. Let us consider three situations $\left\{S^{1}, S^{2}, S^{3}\right\}$ well known by the PMS designer. We consider also the situations $S_{i}^{\text {Good }}, S_{i}^{\text {Neutral }}$ corresponding to the extreme values $P_{\mathrm{i}}=0$ and $P_{\mathrm{i}}=1$. The PMS designer expresses his/her preferences for a given criterion $c_{\mathrm{i}}$ for this set of situations.

$$
S^{\text {Good }} \succ S^{2} \succ S^{1} \succ S^{3} \succ S^{\text {Neutral }}
$$

It means that it is possible to write the following relation between the elementary performances

$$
P_{i}^{\mathrm{Good}} \geq P_{i}^{2}>P_{i}^{1}>P_{i}^{3} \geq P_{i}^{\text {Neutral }} .
$$

Then, he/she expresses his/her strengths of preference as illustrated in figure 2

$$
S^{\text {Good }} \succ^{\text {weak }} S^{2} \succ^{\text {very_strong }} S^{1} \succ^{\text {weak }} S^{3} \succ^{\text {extrem }} S^{\text {Neutral }}
$$

It means that it is possible to write the following relations

$$
\begin{gathered}
P_{i}^{\mathrm{Good}}-P_{i}^{2}=1-P_{i}^{2}=2 \alpha \\
P_{i}^{4}-P_{i}^{1}=5 \alpha \\
P_{\mathrm{i}}^{1}-P_{\mathrm{i}}^{3}=2 \alpha \\
P_{i}^{3}-P_{i}^{\text {Neutral }}=P_{i}^{3}-1=6 \alpha
\end{gathered}
$$

Therefore: $P_{i}^{1} \approx 0.53, P_{i}^{2} \approx 0.87, P_{i}^{3} \approx 0.47(\alpha \approx 0.066)$. (Note that the $\alpha$ coefficient is necessary to respect the interval $[0,1]$ for the performance expression.)

The same procedure of preference elicitation has to be applied for all the criteria. At the end, a set of coherent interval scales is defined. This means that a difference between two situations leading, for example to performances of 0.7 and 0.5 for a criterion of productivity is seen as having an equivalent sense for the PMS designer as a difference between two situations leading to performances of 0.5 and 0.3 . for a criterion of quality.

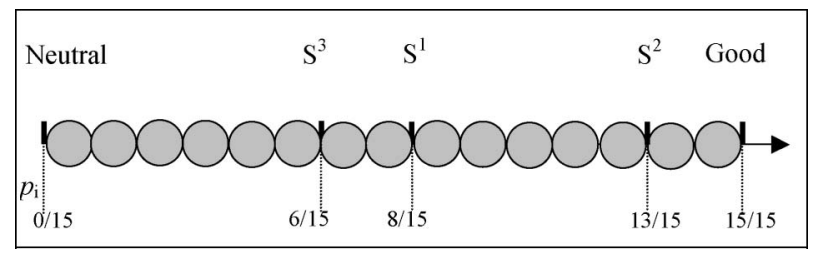

Figure 2. Interval scale illustration. 


\subsection{The aggregated performance expression}

The operators of the CI family are interesting because they include a lot of generalized mean operators (i.e. those included between the min and the max operators). Moreover, they can be written under the form of a conventional WAM modified by effects coming from interactions between elementary performances. More precisely, the 2-additive CI that considers only interactions by pairs is briefly presented hereafter through the 2 following parameters (Brabisch 1997)

(a) the weight of each elementary performance expression in relation to all the other contributions to the overall performance evaluation by the so-called Shapley parameters $v_{\mathrm{i}}^{\prime} s$, that satisfy the condition $\sum_{\mathrm{i}=1}^{\mathrm{n}} v_{\mathrm{i}}=1$, which is a natural condition for decision-makers;

(b) the interaction parameters $I_{\mathrm{ij}}$ of any pair of performance criteria, that range within $[-1,1]$.

In the case of the performance expression, the aggregation formula by the 2-additive CI is given by

$$
P_{\text {overall }}=\sum_{\mathrm{i}=1}^{\mathrm{n}} v_{\mathrm{i}} P_{\mathrm{i}}-\frac{1}{2} \sum_{\mathrm{i} \neq \mathrm{j}}^{\mathrm{n}} I_{\mathrm{ij}}\left|P_{\mathrm{i}}-P_{\mathrm{j}}\right|,
$$

where $\left(P_{1} \ldots, P_{\mathrm{i}} \ldots, P_{\mathrm{n}}\right)$ is the vector of elementary expressions with the condition

$$
\left.v_{\mathrm{i}}-\frac{1}{2} \sum_{\mathrm{j}=1}^{\mathrm{n}}\left|I_{\mathrm{ij}}\right|\right) \geq 0, \forall \mathrm{i} \in[1, \mathrm{n}] \text { et } \mathrm{j} \neq \mathrm{i}
$$

This makes the meanings of $v_{\mathrm{i}}$ and $I_{\mathrm{ij}}$ clearer. Indeed, the overall performance is a weighted mean of the elementary ones modified by terms depending upon the interactions and upon the elementary performance differences. The condition (5) ensures that the overall performance is positive, i.e. the impact of interactions is always inferior to the impact of weights. In order to determine the Shapley parameters $v_{\mathrm{i}}^{\prime} s$ and the interaction coefficients $I_{\mathrm{ij}}{ }^{\prime} s$, the decision-makers need enough aggregated expressions, i.e. as many as the sum of the number of Shapley parameters and the number of interaction coefficients in order to have sufficiently independent equations.

Learning procedures from data are exposed in the literature but in our application context a set of values concerning past situations or resulting from simulations is generally not available during the PMS design process. But the PMS designer is often an experienced manager and thus he/she has expertise concerning the behaviour of the industrial performances. So we propose that he/she considers some particular situations and compares them.
The MACBETH method proposes to consider two types of characteristic situations (possibly fictive):

(a) one elementary expression $P_{\mathrm{i}}$ is good, $P_{\mathrm{i}}=1$ and all the other elementary expressions $P_{\mathrm{j}}$ are bad $P_{\mathrm{j}}=0$. As we have seen before, these situations can be described by elementary expression vectors such as $(0 \ldots, 0,1,0 \ldots, 0)$. In this case, the aggregated performance expression is denoted $P_{\text {overall }}^{\mathrm{i}}$.

(b) one elementary expression $P_{\mathrm{i}}$ is bad, $P_{\mathrm{i}}=0$ and all the other elementary expressions $P_{\mathrm{j}}$ are good, $P_{\mathrm{j}}=1$, these situations can be described by elementary expression vectors such as $(1 \ldots, 1,0,1 \ldots, 1)$. In this case, the aggregated performance expression is noted $P_{\text {overall }}^{\bar{i}}$.

These situations allow the parameters to be derived in a simple way as described in the following example.

Example of CI parameter determination. Let us take a simple example with two criteria $c_{1}$ and $c_{2}$. We then have to determine two Shapley parameters $v_{1}, v_{2}$ and one interaction coefficient $I_{12}$. The expert has to supply information about 4 characteristic situations. It seems natural to consider the situations described from the following performance vectors $(1,1),(1,0),(0,1),,(0,0$,$) and the$ corresponding set of situations $\left\{S^{(1,1)}, S^{(1,0)} S^{(0,1)}, S^{(0,0)}\right\}$.

Firstly, the expert expresses his/her preferences concerning these situations, then he/she provides more knowledge with his/her strengths of preference.

Preferences: $S^{(1,1)} \succ S^{(0,1)} \succ S^{(1,0)} \succ S^{(0,0)}$

It means that it is possible to write the following relation between the aggregated performances:

$$
P_{i}^{(1,1)}>P_{i}^{(0,1)}>P_{i}^{(1,0)}>P_{i}^{(0,0)}
$$

Strengths of preference: $S^{(1,1)} \succ^{6} S^{(0,1)} \succ^{2} S^{(1,0)} \succ^{2} S^{(0,0)}$

This information leads to the following equation system with $v_{\mathrm{i}} \geq 0$ and $-1 \leq I_{i j} \leq+1$ :

$$
\begin{aligned}
& P_{\text {overall }}^{(1,1)}-P_{\text {overall }}^{(0,1)}=6 \alpha=v_{1}+0.5 I_{12} \\
& P_{\text {overall }}^{(0,1)}-P_{\text {overall }}^{(1,0)}=2 \alpha=v_{2}-v_{1} \\
& P_{\text {overall }}^{(1,0)}-P_{\text {overall }}^{(0,0)}=2 \alpha=v_{2}-0.5 I_{12} \\
& P_{\text {overall }}^{(1,1,1)}=v_{1}+v_{2}+v_{3}=1
\end{aligned}
$$

The resolution of the system gives: $v_{1}=0.6 \quad v_{2}=0.4$ $I_{12}=0.4 \alpha=0.1$.

But unfortunately, in our application context, these situations, where almost all the criteria are totally unsatisfied, occur very rarely. Therefore, the PMS designer has only a limited knowledge of these situations and thus 
has difficulties to express his/her preferences. On the opposite, situations where almost all the criteria are satisfied are often encountered because they correspond to a major problem on one particular criterion. The PMS designer is therefore aware of the precise consequences of such problems and can elicit his/her preferences in a precise and reliable way.

Thus, we propose to ask the PMS designers about these situations denoted $S^{\bar{i}}$ instead of the $S^{\mathrm{i}}$ ones. This leads to a little more complex equation resolution as is the case in the application presented in section 4 . Note also that it is possible that no solution with $v_{\mathrm{i}} \geq 0$ and $-1 \leq I_{i j} \leq+1$ is available. It means that the decision maker's preferences are not coherent with a Choquet integral model.

\subsection{Summary of the performance aggregation issue}

Knowing an overall objective and the associated criteria, the quantification of the performance expression can thus be summarized by the following points:

(a) building interval scales for the different identified criteria,

(b) determining the aggregation operator parameters in a consistent way.

In the case of PMS, designers have to use their knowledge to satisfy these two points. So being able to transform their expertise into quantified values is called for. With this in view, we take the MACBETH procedure as a starting point. It allows to quantify both the elementary and the aggregated performances. Then this method, initially designed for the WAM operator, is extended to the 2-additive CI. It will be applied in the case study in section 4 .

\section{Indexes for overall performance understanding}

\subsection{The issue}

Once an overall performance expression has been obtained, the problem is to use it for helping decision-makers increase their understanding about the reasons why a particular level of performance is observed. This leads to answer the following questions: what is the influence of a particular elementary performance on the overall one? and how to improve the overall performance by a minimum increase of the elementary ones? More precisely, let us consider that a situation has led to an elementary performance vector $P^{k}$. Then the overall performance is computed according to an aggregation strategy identified with the Choquet integral operator CI. Ideally, if every thing has proceeded right, we have $A g_{C I}\left(P^{k}\right)=1$ but in practice generally $A g_{C I}\left(P^{k}\right) \neq 1$, and the problem is then to understand why the overall performance is not totally satisfactory, with regard to the elementary ones. The answer to this why question (that can be viewed as an efficacy analysis) can be quantitatively expressed in terms of the relative influence of particular elementary performances on the overall one. A second question is how this result has been obtained versus the means used to reach all the elementary objectives. The answer to this how question (that can be viewed as an efficiency analysis) can be expressed in terms of the means used versus the minimum means required to reach the overall performance.

Let us first consider the case of a weighted mean, i.e. $P_{\text {overall }}=A g_{C I}\left(P^{k}\right)=\sum_{i=1}^{n} a_{i} \cdot P_{i}^{k}$.

Our approach to determine the contribution of elementary performances $P_{j}$ consists simply in defining it as the ratio $a_{j} . P_{j}^{k} / \sum_{i=1}^{n} a_{i} . P_{i}^{k}$. Thus, for a conventional mean (adapted to the Taylorian context), the contribution computation is direct; it is simply equal to the product of the elementary performance by the weight (equal to $a_{i}$ ) of the criterion. This approach can be extended to the Choquet integral (adapted to the post-Taylorian context) by using a linear expression for the Choquet integral, but it is only possible piece-wise as described hereafter.

\subsection{A piece-wise linear expression for the Choquet integral}

As exposed in section 2, the Choquet integral is composed of a linear part that is modified according to mutual interaction between criteria. Going further, Akharraz et al. (2002) have proved that the Choquet integral can be written as a conventional weighted mean in each simplex $H_{\sigma_{k}}=\left\{x \in[0,1] / 0 \leq P_{\sigma(1)}^{k} \leq \ldots \leq P_{\sigma(n)}^{k} \leq 1\right\}$ defined by the ranking of the elementary performances represented by the function $\sigma$. Indeed, for a situation $P^{k}$ belonging to the simplex $H_{\sigma_{k}}$ the Choquet integral has the linear expression: $A g_{C I}\left(P^{k}\right)=\sum_{i=1}^{n} \Delta \mu_{(i)}^{k} \cdot P_{(i)}^{k}$, where $\Delta \mu_{(i)}^{k}=v_{(i)}+\frac{1}{2} \sum_{j>i} I_{(i)(j)}-\frac{1}{2} \sum_{j<i} I_{(j)(i)}$ with $v_{(i)}$ the relative importance of criterion $c_{(i)}$ and $I_{(i)(j)}$ the interaction between criteria $c_{(i)}$ and $c_{(j)}$. Moreover, for every $k: \sum_{i=1}^{n} \Delta \mu_{(i)}^{k}=1$ (the brackets (i) around the indexes indicate the ranking of the criterion $i)$.

To illustrate the preceding formula, let us consider a simple example with 3 criteria to illustrate the idea (an industrial application is presented section 4). We consider $v_{1}=0.4, v_{2}=0.3, v_{3}=0.3$, and $I_{12}=0, I_{13}=0, I_{23}=0.4$. Thus, in this case there are $3 !=6$ different simplexes, but as $I_{12}=0, I_{13}=0$, there are only two different linear parts for the Choquet integral expression: for $\mathrm{P}_{2}<\mathrm{P}_{3}, \Delta \mu_{3}=\Delta \mu_{(3)}=v_{3}-0.5 I_{23}=0.3-0.2=0.1, \Delta \mu_{2}=$ $\Delta \mu_{(2)}=0.3+0.2=0.5, \quad \Delta \mu_{1}=\Delta \mu_{(1)}=0.4 ; \quad$ for $\quad \mathrm{P}_{2}>\mathrm{P}_{3}$, $\Delta \mu_{3}=\Delta \mu_{(2)}=0.5, \quad \Delta \mu_{2}=\Delta \mu_{(3)}=0.1, \quad \Delta \mu_{1}=\Delta \mu_{(1)}=0.4$. Thus, the criteria 2 and 3 have different weights $(0.1$ or 0.5 ) according to the fact that $\mathrm{P}_{2}<\mathrm{P}_{3}$ or $\mathrm{P}_{2}>\mathrm{P}_{3}$; the criterion 1 has a fixed weight of 0.4 . 
In summary, the Choquet integral is a sort of weighted mean with weights $\Delta \mu_{(i)}^{k}$ varying according to the ranking of elementary performances defined by the situation $P^{k}$. Therefore, the extension of the principle described before for the weighted mean can be made as detailed hereafter.

\subsection{Efficacy analysis of the overall performance}

In this sub-section, we propose to consider the partial contribution of a specific elementary performance to the non-performance, i.e. to the fact that the overall performance is not equal to 1 . In fact, this means quantifying the inefficacy of one elementary performance versus the overall inefficacy. The difference $1-A g\left(P^{k}\right)$ can be taken as reflecting the overall inefficacy, and the contribution of one elementary performance to this difference reflects the inefficacy related to this elementary performance.

Using the expression of the preceding paragraph, $\quad 1-\operatorname{Ag}_{C I}\left(P^{k}\right)=\sum_{i=1}^{n} \Delta \mu_{(i)}^{k} .\left(1-P_{(i)}^{k}\right) \quad$ since $\sum_{i=1}^{n} \Delta \mu_{(i)}^{k}=1$ and $\sum_{i=1}^{n} \Delta \mu_{(i)}^{k} \cdot P_{(i)}^{k}=A g_{C I}\left(P^{k}\right)$. Therefore, the inefficacy contribution of the $i$ th elementary performance can be defined by the ratio $\Delta \mu_{(i)}^{k} \cdot\left(1-P_{(i)}^{k}\right) /\left(1-C I\left(P^{k}\right)\right)$. Then, it is possible to rank the different inefficacy contributions in order to exhibit the most important one, that allows conclusions to be made about the reasons of the considered overall inefficacy.

Let us consider again the preceding example with three criteria to illustrate the idea (an industrial application is presented section 4). Thus, in this case there are only two different linear expressions for the Choquet integral: for $P_{2}<P_{3}, \Delta \mu_{1}=0.4, \Delta \mu_{2}=0.5, \Delta \mu_{3}=0.1 ;$ for $P_{2}>P_{3}$, $\Delta \mu_{1}=0.4, \Delta \mu_{2}=0.1, \Delta \mu_{3}=0.5$. Then for a performance vector, $P^{k}=\left(P_{1}^{k}=0.8, P_{2}^{k}=0.8, P_{3}^{k}=0.9\right)$, we obtain $A_{C I}\left(P^{k}\right)=0.81$, The overall inefficacy is $0.19(1-0.81)$, and the elementary performance contributions to this inefficacy are respectively $42.1 \%, 52.6 \%$ and $5.3 \%$. Thus, the most penalizing elementary performance is the one of the criterion 2. Note that if the interactions had not been considered $\left(I_{23}=0\right)$, we would obtain an overall performance of 0.83 and $47 \%, 35.3 \%$ and $17.7 \%$ for the inefficacy contributions. The most penalizing elementary performance would have been the one of the criterion 1 this time.

\subsection{Efficiency analysis of the overall performance}

Another aspect of performance understanding consists in providing evidence concerning the means used to carry out an improvement action aimed at reaching a particular overall performance expression from an initial one, e.g. going from 0.85 to 0.95 (in practice, null elementary performances for all criteria are rarely the initial state). This diagnosis is particularly important when the overall performance is good, because in this case it could seem that everything has gone right: however even when the objectives are reached, decision-makers must still verify that the corresponding means expended by the organization correspond to the planned resources and there has been no waste.

It is worth noticing, that we assimilate here the means to the sum of all the elementary performance variations made to reach the new overall performance. Indeed the means are clearly related to the required performance variation, and for the sake of simplicity we consider only performance increase, the cost aspect of each elementary improvement will be considered in future studies. For example, going from $\quad P^{0}=\left(P_{1}^{0}=0.80, P_{2}^{0}=0.90, P_{3}^{0}=0.85\right)$ to $P^{k}=$ $\left(P_{1}^{k}=0.90, P_{2}^{k}=0.95, P_{3}^{k}=0.90\right)$ could intuitively lead to increase each $P_{i}^{0}$ up to $P_{\mathrm{i}}^{k}$, i.e. to tie up resources such that $\left\|P^{k}-P^{0}\right\|_{1}=\left\|\delta^{k}\right\|_{1}=\sum_{j}\left|\delta_{j}^{k}\right|$ (where $\delta_{j}^{k}$ is the elementary performance increase of criterion $j$ ). Thus in this example $\left\|\delta^{k}\right\|_{1}=(0.90-0.80)+(0.95-0.90)+$ $(0.90-0.85)=0.20$. But as will be seen hereafter, this conventional behaviour at the practical level is not the least costly.

Let us now denote $\Delta^{*}$ the minimum increase of the sum (in the L1-norm sense): $\|\delta\|_{1}=\sum_{i=1}^{n}\left|\delta_{i}\right|$ of the elementary performance increases $\delta_{i}$ that is necessary to go from an initial performance vector $P^{0}=\left(P_{1}^{0}, \ldots, P_{i}^{0}, \ldots, P_{n}^{0}\right)$ to reach a required overall performance in the range $\left[\operatorname{Ag}_{\mathrm{CI}}\left(P^{0}\right), 1\right]$ denoted $P_{\text {overall }}^{*}$. It is thus defined as the optimization problem (P1):

Objective function
$\Delta^{*}=\min \|\delta\|_{1}$
Constraint :
$\operatorname{Ag}_{C I}\left(P_{1}^{0}+\delta_{1}, ., P_{n}^{0}+\delta_{n}\right)=P_{\text {overall }}^{*}$
Bound constraints : $\quad 0 \leq \delta_{i} \leq 1-P_{i}^{0}, \forall i \in\{1, \ldots, n\}$

The details of the increase vector $\delta^{*}=\left(\delta_{1}{ }^{*}, \delta_{2}{ }^{*}, \ldots, \delta_{n}{ }^{*}\right)$ associated to $\Delta^{*}$ are not trivial (see the appendix for details). The solving principle of (P1) is based on the linearity of the Choquet integral in each simplex $H_{\sigma}$ associated to a given ranking of the partial performances. In each $H_{\sigma}$, the (P1) solving problem is reduced to a simplex algorithm. As in practice, the decision-maker only tries to reach an overall expected performance $P_{\text {overall }}^{*}$, the executed elementary increases $\delta_{j}^{\text {observed }}$ are not always optimally performed. Then to define the overall efficiency, we propose to compute the ratio of the minimum increase sum $\Delta^{*}$ w.r.t. the really performed increase sum $\left\|\delta^{\text {observed }}\right\|_{1}: \Delta^{*} /$ $\left\|\delta^{\text {oberved }}\right\|_{1}$ the closer to 1 this ratio, the more efficient the improvement.

Going further in the understanding of performance improvement, we propose to define the efficiency of an elementary performance increase by the difference of the 
observed increase $\delta_{j}^{\text {observed }}$ with the minimal elementary increase contribution denoted $\delta_{j}{ }^{*}$. A positive difference indicates that the partial improvement relative to criterion $j$ is too high and thus not relevant: too many means have been consumed and thus wasted w.r.t. this criterion and over-quality should be diagnosed for criterion $\mathrm{j}$. When the difference is negative, the improvement w.r.t. criterion $\mathrm{j}$ is insufficient and thus indicates that further means should be necessary to achieve this partial goal. Lastly, when the difference is null the way the objective is reached w.r.t. this criterion is optimum.

Let us consider again the preceding example where $P=\left(P_{1}=0.8, \quad P_{2}=0.8, \quad P_{3}=0.9\right)$ and $\operatorname{Ag}_{\mathrm{CI}}(P)=0.81$, and assume that before carrying out the improvement action, the initial performance vector was $P^{0}=$ $\left(P_{1}^{0}=0.6, P_{2}^{0}=0.6, P_{3}^{0}=0.6\right)$ and $\operatorname{Ag}_{\mathrm{CI}}\left(P^{0}\right)=0.6$. The minimum increase vector is $\delta^{*}=\left(\delta_{1}^{*}=0.4, \delta_{2}^{*}=0.08\right.$, $\left.\delta_{3}^{*}=0.08\right)$ with $\Delta^{*}=0.56$ and the observed one is $\delta^{\text {observed }}=\left(\delta_{1}=0.2, \delta_{2}=0.2, \delta_{3}=0.3\right)$ with $\Delta=0.7$, thus the efficiency is $0.56 / 0.7=80 \%$ and the respective efficiencies for the different elementary performances are -0.2 , +0.12 and +0.22 . Thus, the affected means have not been the optimum ones, especially for criteria 3 and 2 for which the means affected have not produced the maximum expected improvement.

\section{Case study}

The case study concerns a SME producing kitchens, bathrooms and storing spaces. The overall objective of the company is to continuously increase the profit margins. In this sense, knowing that the company decides to improve its performance in terms of delay, we consider here more particularly the objective related to the service rate. As stated earlier in this paper, the aim of this study is not to confine decision-makers to a narrow choice of performance indicators to assess and control their processes, but to provide a performance explanatory system, i.e. pieces of information concerning, on the one hand, the reached performances (efficacy) and on the other hand the best way to make a performance improvement (efficiency).

The approach suggested is therefore: first to carry out a top-down objective decomposition, to extract weights, interactions and elementary performances, and then to implement a bottom-up performance aggregation mechanism, and second to compute the proposed indexes for efficacy and efficiency analyses.

\subsection{Objective decomposition and aggregation parameters}

The principle consists in breaking down the top-management strategic objectives according to the various levels of the hierarchical decisional structure of the company. Figure 1 provides a partial decomposition of the strategic objective related to the service rate into tactical objectives and basic criteria to be used to assess operational objectives.

Moreover, according to the production manager's knowledge about the process, the service rate objective is declined into three tactical independent objectives. Applying the Macbeth inspired approach described in section 2 to the tactical performance on time between order and receive leads to the weights indicated in table 1, and to the interactions indicated in table 2.

In summary, the Choquet integral parameters are: $\mathrm{v}_{1}=0.15, \quad \mathrm{v}_{2}=0.4, \quad \mathrm{v}_{3}=0.3, \quad \mathrm{v}_{4}=0.15, \quad$ and $\quad \mathrm{I}_{12}=0.25$, $\mathrm{I}_{13}=0.5, \mathrm{I}_{14}=0.5, \mathrm{I}_{23}=-0.25, \mathrm{I}_{24}=-0.25$, the others being equal to 0 .

\subsection{Efficacy analysis}

Let us consider an objective of 6 weeks for the time between order and receive that has been decomposed into an objective of a 1.5 level for skilfulness, 60 parcels $/ h$ for bottleneck productivity, 0.5 week for work in progress and $95 \%$ for equipment availability. Associated elementary performances have been defined and with the current state, i.e. without designing an action plan, the following elementary performances have been observed $P^{0}=\left(P_{1}^{0}=0.5, P_{2}^{0}=0.5, P_{3}^{0}=0.75, P_{4}^{0}=0.6\right) \quad$ which leads to $A g_{C I}\left(P^{0}\right)=0.55$, i.e. a poor overall performance (a direct indicator gives a value of 0.5 that validates the aggregation model).

Table 1. The weights of the different performance criteria.

\begin{tabular}{lc}
\hline Operational PI & Time between order and receive \\
\hline Skillfulness (1) & 0.15 \\
Bottleneck productivity (2) & 0.35 \\
Work in progress (3) & 0.35 \\
Equipment availability (4) & 0.15 \\
\hline
\end{tabular}

Table 2. The interactions between the different operational criteria.

\begin{tabular}{lcccc}
\hline & Skillfulness & $\begin{array}{c}\text { Bottleneck } \\
\text { productivity }\end{array}$ & $\begin{array}{c}\text { Work in } \\
\text { progress }\end{array}$ & $\begin{array}{c}\text { Equipment } \\
\text { availability }\end{array}$ \\
\hline $\begin{array}{l}\text { Skillfulness } \\
\text { Bottleneck } \\
\text { productivity }\end{array}$ & 0.25 & 0.25 & 0.50 & 0.50 \\
$\begin{array}{l}\text { Work in progress } \\
\text { Equipment }\end{array}$ & 0.50 & -0.25 & & -0.25 \\
$\quad 0.50$ & -0.25 & 0 & 0 \\
$\quad$ availability & & & & \\
\hline
\end{tabular}


Now, it is interesting to understand this poor performance. Applying the method proposed in section 3 gives the following inefficacy indexes: $85 \%, 3 \%, 2 \%$ and $10 \%$. The origin of poor performance is clearly issued in priority from the skilfulness and at a lower level from the equipment availability. This result is quite natural; the link between individual and collective competence and the overall performance being well known in post Taylorian companies. Note that without taking interactions into account, results would be completely different: $18.3 \%, 48.7 \%$, $18.3 \%$ and $14.7 \%$.

\subsection{Efficiency analysis}

According to the objective of 6 weeks for the time between order and receive, an action plan has been designed and implemented. The following new elementary performances were observed $P^{i}=\left(P_{1}^{i}=0.8, P_{2}^{i}=0.6, P_{3}^{i}=0.85\right.$, $\left.P_{4}^{i}=0.7\right)$ which leads to $A g_{C I}\left(P^{i}\right)=0.7$, i.e. an overall improvement of 0.15 for a sum of elementary performance increases of $0.3+0.1+0.1+0.1=0.6$. This sum, that reflects the means used, has to be compared with the minimum sum enabling to reach a value of 0.7 for the overall performance from an initial value of 0.55. Applying the algorithm described in the appendix gives the following minimum increase vector $\delta^{*}=\left(\delta_{1}^{*}=0.19, \delta_{2}^{*}=0.19, \delta_{3}^{*}=0, \delta_{4}^{*}=0.09\right)$ that gives the minimum sum $\Delta^{*}=0.47$. Thus the overall efficiency is $0.47 / 0.6=78.3 \%$. The respective efficiencies for the elementary performances are: $+0.11,-0.09,+0.1,+0.01$. This can be interpreted as too many means affected to Skillfulness and Work In Progress, not enough to the bottleneck productivity, the Equipment availability being correct. This situation can easily be explained by the strategy of the industrial decision-makers which consists in the absolute minimization of the work in progress, in order to reduce the stock costs. Note that these indexes are valid only for a diagnosis analysis, i.e. what has been done, but are not valid for a predictive analysis, i.e. what we have to do next to improve the overall performance. This latter point requires the determination of specific indexes that are currently under development.

\section{Conclusion}

The analysis presented in this article is in keeping with the evolution of industrial performance issues, and specifically with the question of the information to be provided by performance measurement systems. In particular, a characterization of the multi-criteria performance from an approach based on performance aggregation by a Choquet integral has been presented for dealing with criteria interactions. Then the question is to build diagnosis information such that the decision maker better under- stands this more complex performance model. In this view, we have proposed an inefficacy index that quantifies the contribution of one elementary performance to the overall performance inefficacy, and an efficiency index that quantifies the relevance of one elementary performance increase versus the minimal performance increase required to reach the overall one. The next step will concern improvement indexes that quantify the criteria to be improved in priority in order to reach an overall expected performance.

\section{Appendix}

The problem considered is to improve an initial performance vector $P^{0}=\left(P_{1}^{0}, \ldots, P_{i}^{0}, \ldots, P_{n}^{0}\right)$ to a required overall performance in the range $\left[A g_{C I}\left(P^{0}\right), 1\right]$ denoted $P_{\text {overall }}^{*}$ with a minimum value, denoted $\Delta^{*}$, of the sum $\|\delta\|_{1}=\sum_{i=1}^{n}\left|\delta_{i}\right|$ of the elementary performance increases $\delta_{i}$. In fact the problem consists in travelling from one initial point to a higher equi-level curve of the Choquet integral by minimizing the path followed according to the L1-norm. Thus, the problem can be written as the following optimization problem.

The optimization problem (P1) is:

$$
\begin{aligned}
& \text { Objective function } \\
& \Delta^{*}=\min \|\delta\|_{1} \\
& \text { Constraint : } \\
& \operatorname{Ag}_{C I}\left(P_{1}^{0}+\delta_{1}, \ldots, P_{n}{ }^{0}+\delta_{n}\right)=P_{\text {overall }}^{*} \\
& \text { Bound constraints : } \\
& 0 \leq \delta_{i} \leq 1-P_{i}{ }^{0}, \forall i \in\{1, \ldots, n\}
\end{aligned}
$$

It is non linear because of the constraint $\operatorname{Ag}_{C I}\left(P_{1}^{0}+\delta_{1}, . ., P_{n}^{0}+\delta_{n}\right)=P_{\text {overall }}^{*}$. Indeed the Choquet integral is non linear. This appendix provides a method to decompose the problem into $n$ ! simplexes where $A g_{C I}$ is linear $(n !$ is merely the theoretical algorithm complexity but can be efficiently reduced by simple heuristics as proposed below).

Let us consider an initial performance vector $P^{0}$ in the initial simplex domain $H_{\sigma_{0}}$ and corresponding to an initial overall performance $A g_{C I}\left(P^{0}\right)=P_{\text {overall }}^{0}$. The expected overall performance is $P_{\text {overall }}^{*}$. The continuity and monotonicity of the Choquet integral ensure that a performance vector $P^{\delta_{0}} \in H_{\sigma_{0}}$ (i.e. in the same simplex as $P^{0}$, i.e., $P^{0}$ and $P^{\delta_{0}}$ are comonotone) with $\operatorname{Ag}_{C I}\left(P^{\delta_{0}}\right)=P_{\text {overall }^{*}}{ }^{*}$ necessarily exists. (Note that $\forall H_{\sigma_{j}},(1,1, . ., 1) \in H_{\sigma_{j}}$ and $\operatorname{Ag}_{C I}(1,1, . ., 1)=1$.) Firstly, we are searching for this vector by increasing the initial vector performances in a minimum way in the L1 norm sense. In 
this first step, since both performance vectors are in the same simplex $H_{\sigma_{0}}$, the problem to be solved is thus a mere simplex in $H_{\sigma_{0}}$ with:

$$
\begin{aligned}
& \text { Objective function : } \\
& \min \left\|\delta^{0}\right\|_{1} \text { with } \delta^{0}=\left(\delta_{1}^{0}, . ., \delta_{n}^{0}\right) \\
& \text { Constraint : } \\
& A g_{C I}\left(P^{\delta^{0}}\right)=\sum_{q=1}^{n} \Delta \mu_{\sigma_{0}(q)} P_{\sigma_{0}(q)}^{\delta^{0}} \\
& =\sum_{q=1}^{n} \Delta \mu_{\sigma_{0}(q)}\left(P_{\sigma_{0}(q)}^{0}+\delta_{\sigma_{0}(q)}^{0}\right) \\
& =P_{\text {overall }}^{*}
\end{aligned}
$$

These last additional bound constraints enable to rewrite the Choquet Integral as a weighted sum and thus make the problem a linear one in a given simplex $H_{\sigma_{0}}$. In the following, we will see that, as above, the additional bound constraints enable to break down (P1) into several simplexes. Hence, we have found $P^{\delta^{0}}$ with a minimum displacement $\delta^{0}$ in $H_{\sigma_{0}}$ that verifies $A g_{C I}\left(P^{\delta^{0}}\right)=$ $\operatorname{Ag}_{C I}\left(P^{0}+\delta^{0}\right)$. The search of $P^{\delta^{0}}$ is the initial step in the solving of (P1) and let us note the corresponding minimum displacement $\delta^{0}$. But a lower displacement could be obtained by going across other simplexes.

Therefore, we have to consider the search of solutions to (P1) in all the simplexes $H_{\sigma_{j}}$. Let us first consider the minimum displacement from $P^{0}$, denoted $\Delta^{0 \rightarrow j}$, required to reach the border between $H_{\sigma_{0}}$ and $H_{\sigma_{j}}$; the associated border vector is denoted $P^{0 j}$. Then, because of the properties of the Choquet integral mentioned above, we know: $\forall H_{\sigma_{j}}, \exists P^{j}$ such that : $A g_{C I}\left(P^{j}\right)=P_{\text {overall }}^{*}$. Let us denote $\Delta^{0 \rightarrow j}$ the displacement allowing to reach $P_{\text {overall }}^{*}$ by going from the performance vector $P^{0}$ in $H_{\sigma_{0}}$ to the border $P^{0 j}$ and then to the overall expected performance $P^{j}$ in $H_{\sigma_{j}}$ (the displacement from $P^{0 j}$ to $P^{j}$ is denoted $\delta^{j}$ ):

$$
\delta^{0 \rightarrow j}=\left\|\overrightarrow{P^{0} P^{j}}\right\|_{1}=\left\|\overrightarrow{P^{0} P^{0 j}}\right\|_{1}+\left\|\overrightarrow{P^{0 j} P^{j}}\right\|_{1}=\Delta^{0 \rightarrow j}+\delta^{j}
$$

(this equality is due to the use of the L1 norm and $\left.\forall k, \forall j, \delta_{k}^{j} \geq 0\right)$.
We have $A g_{C I}\left(P^{0}+\delta^{0 \rightarrow j}\right)=A g_{C I}\left(P^{0 j}+\delta^{j}\right)=P_{\text {overall }}$ * and we compute $\delta^{j}$ for each $j$, i.e. for each of the $(\mathrm{n} !-1)$ simplexes $H_{\sigma_{j^{\prime}}}$ using the following simplex:

Objective function :

$\min \left\|\delta^{j}\right\|_{1}$ with $\delta^{j}=\left(\delta_{1}^{j}, . ., \delta_{n}^{j}\right)$

Constraint :

$$
\begin{aligned}
A g_{C I}\left(P^{j}\right) & =\sum_{q=1}^{n} \Delta \mu_{\sigma_{j}(q)} P_{\sigma_{j}(q)}^{j} \\
& =\sum_{q=1}^{n} \Delta \mu_{\sigma_{j}(q)}\left(P_{\sigma_{j}(q)}^{0 j}+\delta_{\sigma_{j}(q)}^{j}\right) \\
& =P_{\text {overall }}^{*}
\end{aligned}
$$

Bound constraints :

$$
\mid \begin{aligned}
& 0 \leq \delta_{\sigma_{j}(q)}^{j} \leq 1-P_{\sigma_{j}(q)}^{0 j}, \forall q \\
& P_{\sigma_{j}(q)}^{0 j}+\delta_{\sigma_{j}(q)}^{j} \leq P_{\sigma_{j}(q+1)}^{0 j}+\delta_{\sigma_{j}(q+1)}^{j}, \forall q .
\end{aligned}
$$

Then, we can compute: $\forall j, \delta^{0 \rightarrow j}=\Delta^{0 \rightarrow j}+\delta^{j}$.

Finally we obtain: $\Delta^{*}=\min \left(\left\|\delta^{0}\right\|_{1}, \min \left(\left\|\delta^{0 \rightarrow j}\right\|_{1}\right)\right)$.

The linear problems in $H_{\sigma_{0}}$ or in $H_{\sigma_{j}}$ are exactly identical but a particular attention is paid to the search in $H_{\sigma_{0}}$ : indeed, when $\delta^{0}$ is available, as soon as $\Delta^{0 \rightarrow j}>\delta^{0}$, no search in $H_{\sigma_{j}}$ needs to be done. As a consequence, although the theoretical complexity of the algorithm is $n !$, simple heuristics like that enable to reduce it significantly.

\section{References}

Akharraz, A., Mauris, G. and Montmain, J., A project decision support system based on an elucidative fusion system. Fith International Conference on Information Fusion (ICIF 02), Annapolis, USA, pp. 593-599, July 2002.

Bana e Costa, C.A. and Vansnick, J.C., Applications of the MACBETH approach in the framework of an additive aggregation model. J. MultiCriteria Dec. Analysis, 1997, 6, 107-114.

Bana e Costa, C.A., De Corte, J.M. and Vansnick, J.C., On the mathematical foundations of Macbeth. In MCDA. Multiple Criteria Decision Analysis, edited by J. Figueira, S. Greco and M. Ehrgott, pp. 409-442, 2004 (Kluwer Academic Publishers: Dordrecht, The Netherlands).

Berrah, L., Mauris, G. and Foulloy, L., The aggregation of industrial performance information by the Choquet fuzzy integral. Soft Computing in Measurement and Information Acquisition, edited by L. Reznik and V. Kreinovich, pp. 121-135, 2003 (Physica-Verlag).

Berrah, L., Mauris, G., Foulloy, L. and Haurat, A., Global vision and performance indicators for an industrial improvement approach. Comput. Ind., 2000, 43, 211-225.

Bititci, U.S., Modelling of performance measurement systems in manufacturing enterprises. Int. J. Prod. Econ., 1995, 42, 137-147.

Bradford, J. and Childe, S.J., A non-linear redesign methodology for manufacturing systems. International Conference on Stimulating Manufacturing Excellence in Small and Medium Enterprises (SMESME'01), Aalborg, Denmark, 14-16 May 2001, pp. 52-59. 
Cha, Y. and Jung, M., Satisfaction assessment of multi-objective schedules using neural fuzzy methodology. Int. J. Prod. Res., 2003, 41, 1831-1849.

Clivillé, V., Berrah, L. and Mauris, G., Information fusion in industrial performance: a 2-additive Choquet integral based approach. IEEE International Conference on Systems, Man and Cybernetics (SMC 04), CD-ROM , La Haye, Pays-Bas, October 2004.

Fortuin, L., Performance indicators - why, where and how? Eur. J. Opl Res., 1988, 34, 1-9.

Ghalayini, A.M., Noble, J.S. and Crowe, T.J., An integrated dynamic performance measurement system for improving manufacturing competiveness. Int. J. Ops Prod. Mgmt., 1997, 15, 80-116.

Grabisch, M. and Roubens, M., The application of fuzzy integrals in multicriteria decision making. Eur. J. Opl Res., 1996, 89, 445-456.

Grabisch, M., $k$-ordered discrete fuzzy measures and their representation. Fuzzy Sets Systems, 1997, 92, 167-189.

Grabot, B., Objective satisfaction assessment using neural nets for balancing multiple objectives. Int. J. Prod. Res., 1998, 36, 2377-2395.

Kaplan, R.S. and Norton, D.P., The balanced scorecard: measures that drives performance. Harvard Bus. Rev., January-February, 1992.

Kueng, P. and Krahn, A.J., Building a process performance measurement system: some early experiences. J. Scientific Ind. Res., 1999, 58, 149-159.

Labreuche, C. and Grabisch, M., The Choquet integral for the aggregation of interval scales in multi-criteria decision making. Fuzzy Sets and Systems, 2003, 137, 11-26.
Lebas, M.J., Performance measurement and performance management. Int. J. Prod. Econ., 1995, 41, 23-35.

Montmain, J., Denguir, A. and Tardy, J., An interactive decision-making support system for organization management and diagnosis. Modeling, Computation and Optimization in Information Systems and Management Sciences, Metz, France, 2004.

Neely, A., The performance measurement revolution: why now and what next. Int. J. Ops Prod. Mgmt., 1999, 19, 205-228.

Rangone, A., An analytical hierarchy process framework for comparing the overall performance of manufacturing departments. Int. J. Ops Prod. Mgmt., 1996, 16, 104-119.

Rangone, A., An analytical hierarchy process framework for comparing the overall performance of manufacturing departments. Int. J. Ops Prod. Mgmt., 1996, 16, 104-119.

Saaty, T., The analytic hierarchy and the analytic network processes for the measurement of intangible criteria and for decision-making. In $M C D A$. Multiple Criteria Decision Analysis, edited by J. Figueira, S. Greco and M. Ehrgott, pp. 345-407, 2004 (Kluwer Academic Publishers: Dordrecht, The Netherlands).

Suwignjo, P. and Bititci, U.S., Quantitative models for performance measurement system. Int. J. Prod. Econ., 2000, 64, 231-241. 